Article

\title{
Acoustic Localization for a Moving Source Based on Cross Array Azimuth
}

\author{
Junhui Yin ${ }^{1}$, Chao Xiong ${ }^{1}$ and Wenjie Wang ${ }^{2, *}$ \\ 1 Department of Artillery Engineering, Army Engineering University, Shijiazhuang 050003, China; \\ yuanzhidao@163.com (J.Y.); xiongchao@tsinghua.org.cn (C.X.) \\ 2 Fluid and Acoustic Engineering Laboratory, Beihang University, Beijing 100191, China \\ * Correspondence: wangwenjie@buaa.edu.cn; Tel.: +86-108-231-7407
}

Received: 5 June 2018; Accepted: 28 July 2018; Published: 1 August 2018

Featured Application: the work introduced in this paper can be used for wildlife conservation, health protection, and other engineering applications.

\begin{abstract}
Acoustic localization for a moving source plays a key role in engineering applications, such as wildlife conservation and health protection. Acoustic detection methods provide an alternative to traditional radar and infrared detection methods. Here, an acoustic locating method of array signal processing based on intersecting azimuth lines of two arrays is introduced. The locating algorithm and the precision simulation of a single array shows that such a single array has good azimuth precision and bad range estimation. Once another array of the same type is added, the moving acoustic source can be located precisely by intersecting azimuth lines. A low-speed vehicle is used as the simulated moving source for the locating experiments. The length selection of short correlation and moving path compensation are studied in the experiments. All results show that the proposed novel method locates the moving sound source with high precision $(<5 \%)$, while requiring fewer instruments than current methods.
\end{abstract}

Keywords: acoustic localization; cross array; moving sound source; discrete sampling; error analysis

\section{Introduction}

The localization of moving sources represents a major issue in engineering applications. Similar to other detection technologies, acoustic-localization methods have been developed rapidly over the years. Meanwhile, the noise generated by low-speed vehicles (LPVs) is a key issue, especially in connection with acoustics mitigation, where noise pollution continues to be a major health problem, with a whole host of health effects, such as: sleep disorders with awakenings [1], learning impairment [2,3], hypertension ischemic heart disease [4], and especially annoyance [5], a widely used indicator to study the effect of different noise sources on wellbeing. In this context, the main effort has been done to mitigate the main sources of noise: road traffic [6-8], railway traffic [9,10], airport [11,12], and industrial [13]. Specifically about road noise, the most important interaction producing noise, more than just the engine noise used for the LPV, was also road/tire interaction $[14,15]$ and aerodynamic noise for high-speed vehicles. Furthermore, a relatively new noise source is impacting modern society in areas where background noise is low. Wind farms are being installed continuously every year to supply energy demand, but people are being affected by its noise, which is more disturbing than other sources $[16,17]$ and the scientific community is moving towards its assessment [18].

In this paper, the LPV is the research object. As for all moving vehicles, exhaust systems and chain tracks are the main noise sources of LPVs, with exhaust systems representing the dominant factor. Therefore, exhaust systems could be chosen as the moving noise source. The most common 
localization methods for noise sources are Nearfield Acoustic Holography (NAH), beamforming, and array signal processing [19]. The sound field of a moving vehicle is effectively measured based on NAH with a moving acoustic plane [20] and coordinate compensation [21,22]. Far-field measurements of a moving source can be achieved by the short-time beamforming method, but these require extensive computational resources for processing the acquired data for the acoustic plane frame at every moment. The false noise source (ghost image) would also be easily generated $[23,24]$. For array signal processing, the required computations are fast and can be performed to high precision $[25,26]$. This is so since the necessary calculations to be performed on the signals are only one-dimensional and, therefore, substantially less demanding than those for a whole acoustic plane.

The localization method of a moving sound source for the new method described here is achieved by intersecting the azimuth lines of cross arrays. Initial testing of the localization algorithm and the data analysis were performed for a single array and revealed a good performance. Therefore, a second array was added to cross the azimuth lines. The locating experiments were conducted with the engine noise of an LPV as a moving noise source. The data length determined by short-time correlation and path compensation were also introduced. The new method succeeded in effective localization of moving vehicles, requiring less expensive instrumentation than existing methods. Moreover, it was found that it continues to perform properly even under adverse ambient conditions, such as bad weather or at low light levels at night.

\section{Localization Analysis of Single Array}

\subsection{Localization Algorithm}

The LPV used for the current study travelled on level ground such that its height remained constant relative to the array sensors. The height of the vehicle was about $2.0 \mathrm{~m}$, which was approximately $1.5 \mathrm{~m}$ higher than the arrays themselves. Compared to the range of about $100 \mathrm{~m}$ or more, the constant height difference between the vehicle and the arrays had little influence on the localization performance and accuracy. Therefore, the localization was operated in the $x-y$ coordinate system while height difference was ignored. The five-element cross array was taken as a basic array pattern, as illustrated in Figure 1.

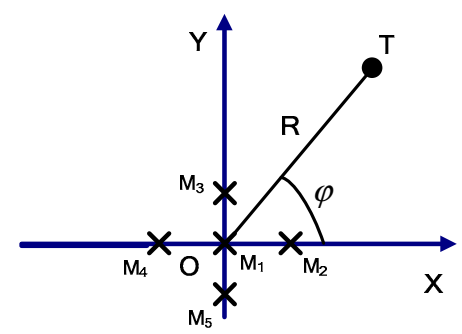

Figure 1. Model of single array.

The coordinates are defined within the plane of the array. The center acoustic sensor is located at $O(0$, 0 ), while the remaining four were $M_{2}(\mathrm{D}, 0), M_{3}(0, \mathrm{D}), M_{4}(-\mathrm{D}, 0)$, and $M_{5}(0,-\mathrm{D})$, where $\mathrm{D}$ represents the distance from $M_{i}$ to $O$. The noise source is assumed to be located at $T(x, y)$, with an angle $\varphi$ between $O T$ and $x$ axis as indicated in Figure 1. The time delays between the arrival time of noise at the center sensor and the other four sensors are referred to as $\tau_{1 i}$. Similarly, $d_{1 \mathrm{i}}(i=2,3,4,5)$ represents the distance between the center sensor and the neighboring sensors, such that $d_{1 \mathrm{i}}=\mathrm{c} \times \tau_{1 \mathrm{i}}$ ( $c$ is current sound velocity). $\mathrm{R}$ is the distance from $O$ to $T$. 
From the simple geometry in Figure 1, the distances can be expressed.

$$
\left\{\begin{array}{l}
x^{2}+y^{2}=R^{2} \\
(x-D)^{2}+y^{2}=\left(R+d_{12}\right)^{2} \\
x^{2}+(y+D)^{2}=\left(R+d_{13}\right)^{2} \\
(x+D)^{2}+y^{2}=\left(R+d_{14}\right)^{2} \\
x^{2}+(y-D)^{2}=\left(R+d_{15}\right)^{2}
\end{array}\right.
$$

The solution of Equation (1) is

$$
\begin{gathered}
\left\{\begin{array}{l}
x=\frac{2 R\left(d_{14}-d_{12}\right)+d_{14}^{2}-d_{12}^{2}}{2 D} \\
y=\frac{2 R\left(d_{13}-d_{15}\right)+d_{13}^{2}-d_{15}^{2}}{2 D}
\end{array}\right. \\
\left\{\begin{array}{l}
\tan \varphi=\frac{y}{x}=\frac{\left(\tau_{15}-\tau_{13}\right)\left[2 R-\mathrm{c}\left(\tau_{15}-\tau_{13}\right)\right]}{\left(\tau_{14}-\tau_{12}\right)\left[2 R-c\left(\tau_{11}-\tau_{12}\right)\right]} \\
R=\sqrt{x^{2}+y^{2}}=\frac{4 D^{2}-d_{12}^{2}-d_{13}^{2}-d_{14}^{2}-d_{15}^{2}}{2\left(d_{12}+d_{13}+d_{14}+d_{15}\right)}
\end{array}\right.
\end{gathered}
$$

when $R>c \times \tau_{1 i}$,

$$
\tan \varphi \approx \frac{\left(\tau_{15}-\tau_{13}\right)}{\left(\tau_{14}-\tau_{12}\right)}
$$

then Equation (3) can be simplified:

$$
\left\{\begin{array}{l}
\varphi=\arctan \frac{\left(\tau_{15}-\tau_{13}\right)}{\left(\tau_{14}-\tau_{12}\right)} \\
R=\left(4 D^{2}-c^{2} \sum_{i=2}^{5} \tau_{1 i}{ }^{2}\right) / 2 c \sum_{i=2}^{5} \tau_{1 i}
\end{array}\right.
$$

The location of the noise source is given by Equations (2) and (5) and, with reference to their derivation, it is evident that the localization algorithm is based on the time delays between the arrival times of noise at the sensors in the array.

\subsection{Precision Analysis for Localization}

The algorithm for localizing the noise source, as described by Equations (2) and (5) in Section 2.1, and the associated accuracy depend on sound velocity $c$, array size $D$ and, in particular, the error involved in estimating the time delay $\sigma_{\tau}$. Since $D$ and $c$ remain constant, for any particular array and measurement environment, the dominant factor affecting the precision of the proposed method is associated with the error involved in measuring $\sigma_{\tau}$. Due to the symmetric arrangement of the sensors with regard to the central sensor, the standard errors for the time delay of all sensors were assumed to be equal, such that $\sigma_{\tau}=\sigma_{\tau 1 i}$.

In Equation (5), quadratic function was included in the expression of coordinates $(x, y)$, which makes it different to calculate the transmission. Then, after the precision calculation of coordinates was transferred into angular coordinates, the localization was described with azimuth $\varphi$ and range $R$ as illustrated in Equation (5).

\subsection{Azimuth Precision}

According to Equation (5), azimuth $\Phi$ was a function of time delay $\tau$.

$$
\Phi=\mathrm{F}(\tau)=\mathrm{F}\left(\tau_{12}, \tau_{13}, \tau_{14}, \tau_{15}\right)
$$

The transmission form of azimuth error $\sigma_{\varphi}$ can be expressed.

$$
\sigma_{\varphi}^{2}=\left(\frac{\partial \varphi}{\partial \tau_{12}} \sigma \tau\right)^{2}+\left(\frac{\partial \varphi}{\partial \tau_{13}} \sigma \tau\right)^{2}+\left(\frac{\partial \varphi}{\partial \tau_{14}} \sigma \tau\right)^{2}+\left(\frac{\partial \varphi}{\partial \tau_{15}} \sigma \tau\right)^{2}
$$


Taking derivative of $\tau$ in Equation (5):

$$
\left\{\begin{array}{l}
\frac{\partial \varphi}{\partial \tau_{12}}=-\frac{\partial \varphi}{\partial \tau_{14}}=\frac{1}{1+\tan ^{2} \varphi} \cdot \frac{\tau_{15}-\tau_{13}}{\left(\tau_{14}-\tau_{12}\right)^{2}} \\
\frac{\partial \varphi}{\partial \tau_{13}}=-\frac{\partial \varphi}{\partial \tau_{15}}=-\frac{1}{1+\tan ^{2} \varphi} \cdot \frac{1}{\tau_{14}-\tau_{12}}
\end{array}\right.
$$

So the expression of azimuth error $\sigma_{\varphi}$ is

$$
\sigma_{\varphi}=\frac{\sigma \tau}{1+\tan ^{2} \varphi} \sqrt{\frac{2\left(\tau_{14}-\tau_{12}\right)^{2}+2\left(\tau_{15}-\tau_{13}\right)^{2}}{\left(\tau_{14}-\tau_{12}\right)^{4}}}
$$

Solving Equations (2) and (5):

$$
\left\{\begin{array}{l}
\left(\tau_{14}-\tau_{12}\right)^{2}+\left(\tau_{15}-\tau_{13}\right)^{2}=\frac{D^{2}}{v^{2}} \\
\left(\tau_{14}-\tau_{12}\right)^{2}=\frac{D^{2}}{v^{2}\left(1+\tan ^{2} \varphi\right)}
\end{array}\right.
$$

Substituting Equation (10) into Equation (9):

$$
\sigma_{\varphi}=\frac{\partial \varphi}{\partial \tau_{i}}=\frac{\sqrt{2} c}{D} \sigma_{\tau}
$$

Thus, the azimuth error is determined by $c, D$, and $\sigma_{\tau}$. We assume a value of $c=343 \mathrm{~m} / \mathrm{s}$ for the sound velocity and employ a sampling rate of $5000 \mathrm{~Hz}$. The sampling interval is $200 \mu$ s and the distribution of azimuth error is shown in Figure 2.

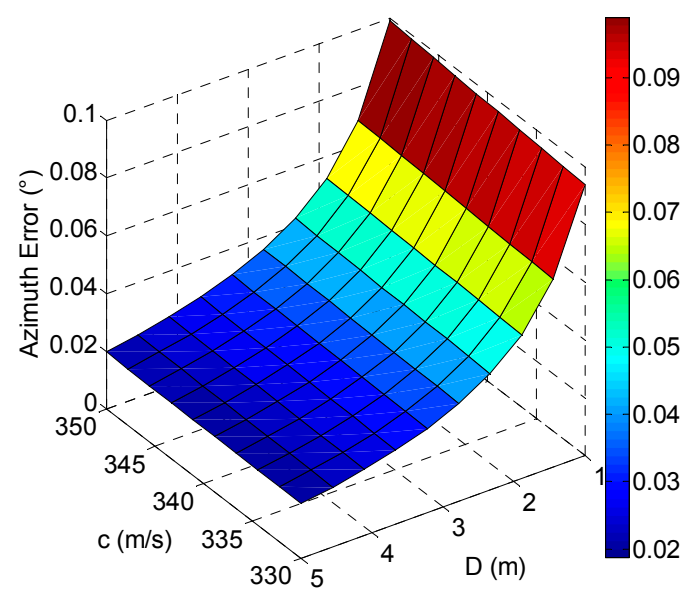

(a) $\sigma_{\tau}=100 \mu \mathrm{s}$

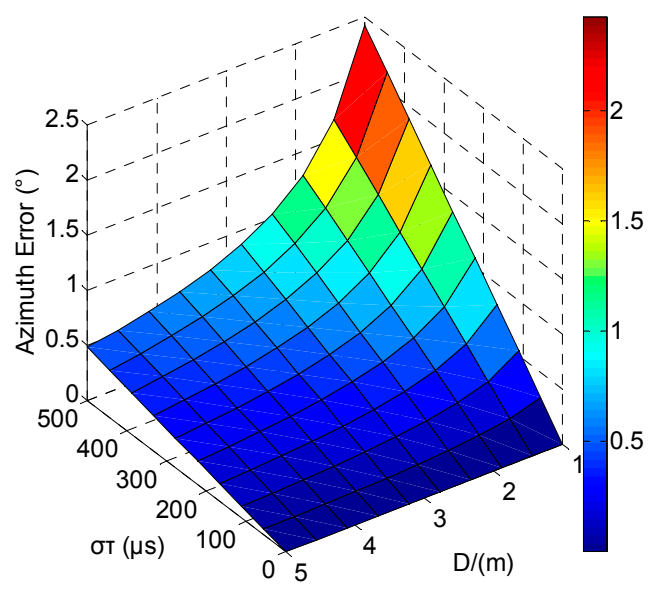

(b) $c=343 \mathrm{~m} / \mathrm{s}$

Figure 2. Distribution of azimuth error of $c$ and $\sigma_{\tau}$.

Figure 2 shows that the relationship of $\sigma_{\varphi}$ and $c$ was linear, as well as $\sigma_{\tau}$. However, the one between $\sigma_{\varphi}$ and $D$ was inverse. In the condition of $D \geq 2 \mathrm{~m}, \sigma_{\varphi}$ stays at an optimal level as $0.03^{\circ}$ in Figure 2a; when $\sigma_{\tau}=100 \mu \mathrm{s}$ in Figure $2 \mathrm{~b}$ it stays $0.1^{\circ}$ when $c$ was set as $343 \mathrm{~m} / \mathrm{s}$.

\subsection{Range Precision}

The range is also a function of time delay $\tau$, and the transmission error is:

$$
\sigma_{R}^{2}=\left(\frac{\partial R}{\partial \tau_{12}} \sigma \tau\right)^{2}+\left(\frac{\partial R}{\partial \tau_{13}} \sigma \tau\right)^{2}+\left(\frac{\partial R}{\partial \tau_{14}} \sigma \tau\right)^{2}+\left(\frac{\partial R}{\partial \tau_{15}} \sigma \tau\right)^{2}
$$


Evaluating the partial derivatives of $\tau$ in Equation (5) gives:

$$
\frac{\partial R}{\partial \tau_{1 i}}=\left[2 c^{2} \tau_{1 i} \sum_{j=2}^{5} \tau_{1 j}-\left(c^{2} \sum_{j=2}^{5} \tau_{1 j}{ }^{2}-4 D^{2}\right)\right] / 2 c\left(\sum_{j=2}^{5} \tau_{1 j}{ }^{2}\right.
$$

Substituting Equation (5) into Equation (13) yields:

$$
\frac{\partial R}{\partial \tau_{1 i}}=\left(c \tau_{1 i}-R\right) / \sum_{j=2}^{5} \tau_{1 j}
$$

According to the geometric relation of array and target:

$$
\begin{aligned}
\tau_{1 \mathrm{i}} & =\frac{1}{\mathrm{c}}\left\{\mathrm{R}-\sqrt{\mathrm{R}^{2}+\mathrm{D}^{2}-2 \mathrm{RD} \cos \left[\varphi-(\mathrm{i}-1) \frac{\pi}{2}\right]}\right\} \\
& =\frac{R}{c}-\frac{R}{c} \sqrt{1+\left[\frac{D}{R}\right]^{2}-2\left[\frac{D}{R}\right] \cos \left[\varphi-(\mathrm{i}-1) \frac{\pi}{2}\right]}
\end{aligned}
$$

then the Taylor expansion of Equation (15) is:

$$
\begin{aligned}
& \tau_{1 \mathrm{i}}=\frac{R}{v}-\frac{R}{v}\left\{1+\frac{1}{2}\left[\left(\frac{D}{R}\right)^{2}-2\left(\frac{D}{R}\right) \cos \left[\varphi-(\mathrm{i}-1) \frac{\pi}{2}\right]\right\}\right. \\
& -\frac{1}{8}\left\{\left(\frac{D}{R}\right)^{2}-2\left(\frac{D}{R}\right) \cos ^{2}\left[\varphi-(\mathrm{i}-1) \frac{\pi}{2}\right]\right\} \\
& \approx \frac{R}{v}\left\{\frac { 1 } { 2 } \left[\left(\frac{D}{R}\right)^{2}-\left(\frac{D}{R}\right) \cos \left[\varphi-(\mathrm{i}-1) \frac{\pi}{2}\right]\right.\right. \\
& \left.-\frac{1}{2}\left(\frac{D}{R}\right)^{2} \cos ^{2}\left[\varphi-(\mathrm{i}-1) \frac{\pi}{2}\right]\right\} \\
& \sum_{i=2}^{5} \tau_{1 i}=-\frac{2 D^{2}}{R c}+\frac{D}{c} \sum_{i=2}^{5} \cos \left[\varphi-(i-1) \frac{\pi}{2}\right] \\
& +\frac{D^{2}}{2 R c} \sum_{i=2}^{5} \cos ^{2}\left[\varphi-(i-1) \frac{\pi}{2}\right] \\
& \sum_{\mathrm{i}=2}^{5} \tau_{1 \mathrm{i}} \approx-\frac{2 \mathrm{D}^{2}}{\mathrm{Rc}}
\end{aligned}
$$

and then substituting Equation (18) into Equation (14) yields:

$$
\frac{\partial R}{\partial \tau_{1 i}} \approx-\frac{2 R c\left(c \tau_{1 i}-R\right)}{3 D^{2}}
$$

Therefore, Equation (14) then becomes

$$
\sigma_{R}=\frac{4 R c \sqrt{D^{2}+R^{2}}}{3 D^{2}} \sigma_{\tau}
$$

Equation (20) reveals that the range error $\sigma_{R}$ is determined by range $R$, array size $D$, sound velocity $c$, and the delay error. Compared to the azimuth error, the influence of $R$ here is an additional effect on the error. Assuming values of $5 \mathrm{kHz}$ for the sampling rate, $R=100 \mathrm{~m}$, and array size changed from $1 \mathrm{~m}$ to $5 \mathrm{~m}$, the distribution of range error is shown in Figure 3. 


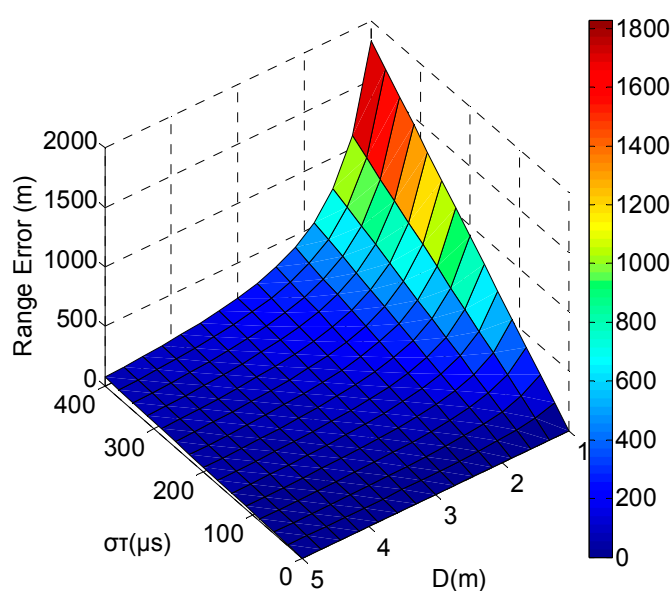

(a) $\sigma_{\tau}=100 \mu \mathrm{s}$

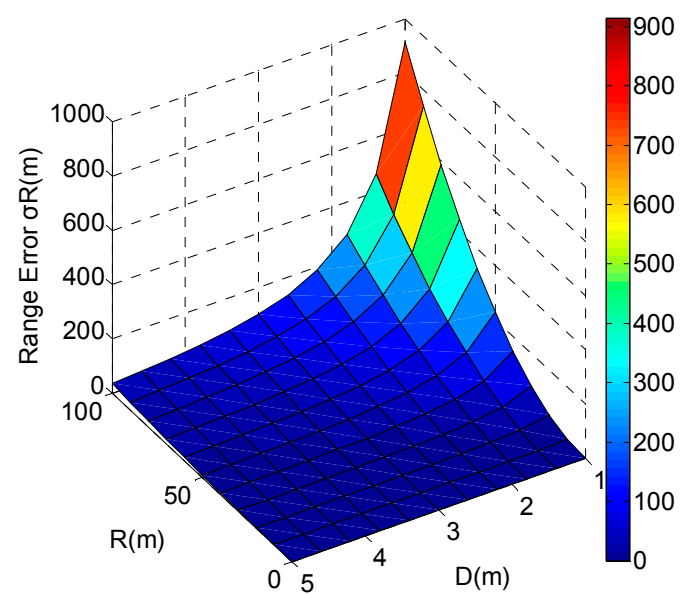

(b) $R=100 \mathrm{~m}$

Figure 3. Distributions of the range error of $R$ and $\sigma_{\tau}$.

In Figure 3, the range error was quite big. In both cases, the error reduces with increasing array size, and it increases with both the range $R$ and the time delay $\sigma_{\tau}$. In general, however, the error is overall at a fairly high level. In Figure 3 a, the relative error was almost $40 \%$ under the optimal condition, and the biggest error was $950 \%$. The error distribution in Figure $3 b$ is the same as in Figure $3 a$, with higher level, the optimal error was $50 \%$, and the biggest one is twenty times.

In summary, a single five-element cross array has good directional ability. The azimuth error can stay below $0.1^{\circ}$ under reasonable conditions. However, the range ability is rather bad. The error is nearly $40 \%$ even under best conditions, which makes it impossible to achieve satisfactory sound source localization.

\section{Localization Analysis of Double Arrays}

\subsection{Localization Principle}

Although the single array has poor range-detection ability, its good directional ability ensures that the direction of the sound source is accurately determined. In order to improve the range-detection ability, a second array was added to the setup by means of intersecting the azimuth lines.

The array in Figure 1 remained positioned as shown in the figure and is referred to as Array 1. The second array, with identical characteristics, was added to the $X-Y$ plane as Array 2 . The centre of Array 2 is located at $O_{1}(L, 0)$. The angle between the line $O T$ (sound source $T$ to origin $O$ ) and the axis-X is referred to as $\varphi_{1}$, while the angle between $O_{1} T$ and $\mathrm{X}$ is $\varphi_{2}$. The time delay when the sound signal reaches the sensors in Array 2 is $\tau_{1 i}{ }^{\prime}$. The range differences are $d_{1 i}{ }^{\prime}(i=2,3,4,5)$, so $d_{1 i}{ }^{\prime}=c \times \tau_{1 i}{ }^{\prime}$. The geometry of the double-array setup is shown in Figure 4.

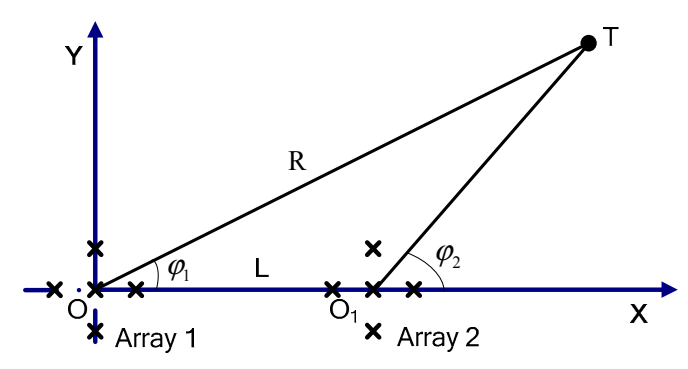

Figure 4. Model of double array. 
Since the structure of both arrays is the same, the form of the azimuth formula is the same and the relevant expressions follow from Equation (3) as:

$$
\left\{\begin{aligned}
\tan \varphi_{1} & \approx \frac{\left(\tau_{15}-\tau_{13}\right)}{\left(\tau_{11}-\tau_{12}\right)} \\
\tan \varphi_{2} & \approx \frac{\left(\tau_{15^{\prime}}-\tau_{13^{\prime}}\right)}{\left(\tau_{14^{\prime}}-\tau_{12^{\prime}}\right)}
\end{aligned}\right.
$$

From the geometric relationship,

$$
\left\{\begin{array}{l}
k_{1}=\frac{y}{x}=\tan \varphi_{1} \\
k_{2}=\frac{y}{x-L}=\tan \varphi_{2}
\end{array}\right.
$$

The simplification of Equation (22) is:

$$
\begin{gathered}
\left\{\begin{array}{l}
x=\frac{L k_{2}}{k_{2}-k_{1}} \\
y=\frac{L k_{1} k_{2}}{k_{2}-k_{1}}
\end{array}\right. \\
\left\{\begin{array}{l}
\varphi=\arctan \left(k_{1}\right) \\
R=\frac{L k_{2} \sqrt{1+k^{2}}}{k_{2}-k_{1}}
\end{array}\right.
\end{gathered}
$$

Equations (23) and (24) represent two alternative expressions for the localization of sound source. In these two expressions, the variables are array distance $L$ and slopes $k_{1}$ and $k_{2}$. The slopes can be inferred from the time delay at each one of the two sensors by means of Equation (21). In the experiments, localization was obtained from time delay $\tau_{1 i}{ }^{\left({ }^{\prime}\right)}$ and array distance $L$.

\subsection{Precision Analysis for Localization}

Compared to the single array, the variable $L$ has been added to the localization expression for double arrays. However, the time delay remains the key variable. As the structure and sensors of the two arrays are identical, the standard time delay errors of both are equal $\left(\sigma_{\tau}=\sigma_{\tau 12}=\sigma_{\tau 13}=\sigma_{\tau 14}=\right.$ $\left.\sigma_{\tau 15}=\sigma_{\tau 12}^{\prime}=\sigma_{\tau 13}^{\prime}=\sigma_{\tau 14}^{\prime}=\sigma_{\tau 15}^{\prime}\right)$.

As the direction was determined by Array 1, the azimuth error was analyzed according to Equation (24). Meanwhile, range error $\sigma_{R}$ is influenced by azimuth error $\sigma_{\varphi}$ and array distance $L$. Range precision is obviously determined by the azimuth precision according to Equation (24). Range precision can be expressed with error transmission as

$$
\begin{gathered}
\sigma_{R}=\frac{\partial R}{\partial \tau_{i}}=\frac{\partial R}{\partial \varphi} \frac{\partial \varphi}{\partial \tau_{i}}=\left(\frac{\partial R}{\partial \varphi_{1}}+\frac{\partial R}{\partial \varphi_{2}}\right) \frac{\partial \varphi}{\partial \tau_{i}} \\
\sigma_{R}=\frac{\sqrt{2} c r\left(\sin \left(\varphi_{1}+\varphi_{2}\right)+\sec \varphi_{1} \cos \varphi_{2}\right)}{\sin \left(\varphi_{1}-\varphi_{2}\right)} \sigma_{\tau}
\end{gathered}
$$

Applying the sin theorem on $\Delta \mathrm{TO}_{1} \mathrm{O}_{2}$ gives:

$$
\begin{gathered}
\frac{\sin \angle T O_{2} O_{1}}{R}=\frac{\sin \angle O_{2} T O_{1}}{\mathrm{~L}} \\
\frac{\sin \varphi_{2}}{R}=\frac{\sin \left(\varphi_{2}-\varphi_{1}\right)}{\mathrm{L}} \\
R=\frac{\mathrm{L} \sin \varphi_{2}}{\sin \left(\varphi_{2}-\varphi_{1}\right)}
\end{gathered}
$$

Taking partial derivative in Equation (29) yields:

$$
\sigma_{R}=\frac{\sqrt{2} c L \cos \varphi_{2}}{D \sin \left(\varphi_{2}-\varphi_{1}\right)} \sigma_{\tau}
$$


Substituting in Equation (29) gives

$$
\sigma_{R}=\frac{\sqrt{2} c R}{D \tan \varphi_{2}} \sigma_{\tau}
$$

In Equation (31), range precision is determined by sound velocity $c$, array size $D$, azimuth $\varphi_{2}$ of Array 2, error of time delay $\sigma_{\tau}$, and range $R$. The distribution of the range error is shown in Figure 5 . In Figure $5 \mathrm{a}, R=100 \mathrm{~m}, c=343 \mathrm{~m} / \mathrm{s}, \sigma_{\tau}=100 \mu \mathrm{s}$. In Figure $5 \mathrm{~b}, D=3 \mathrm{~m}, R=100 \mathrm{~m}, c=343 \mathrm{~m} / \mathrm{s}$.

Figure 5 reveals that $\varphi_{2}$ affected range precision substantially. In $\left(1^{\circ}, 20^{\circ}\right)$ and $\left(160^{\circ}, 179^{\circ}\right)$, the range error remains very high. In $\left(20^{\circ}, 160^{\circ}\right)$, the error was much lower and acceptable. In Figure $5 \mathrm{a}$, array size $D$ significantly affected precision when $D<3 \mathrm{~m}$. Error was $10.78 \%$ when $\varphi_{2}$ was $8.12^{\circ}$. The error reduced as angle $\varphi_{2}$ is increased. When $\varphi_{2}$ was $15.24^{\circ}$, range error was $5.65 \%$ and it reduced to $3.78 \%$ as $\varphi_{2}$ was increased to $22.36^{\circ}$. In Figure $5 b$, distribution was the same to Figure $5 a$, and the error stayed below $5 \%$ when $\varphi_{2}$ is above $20^{\circ}$.

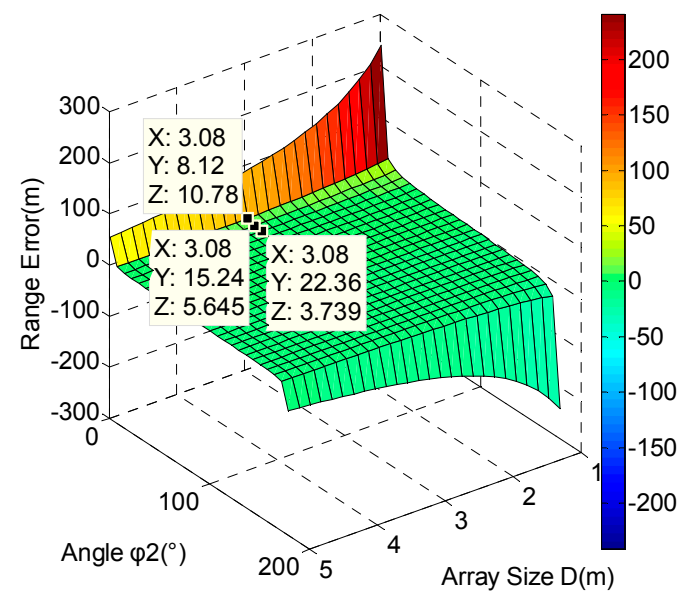

(a) $R=100 \mathrm{~m}$

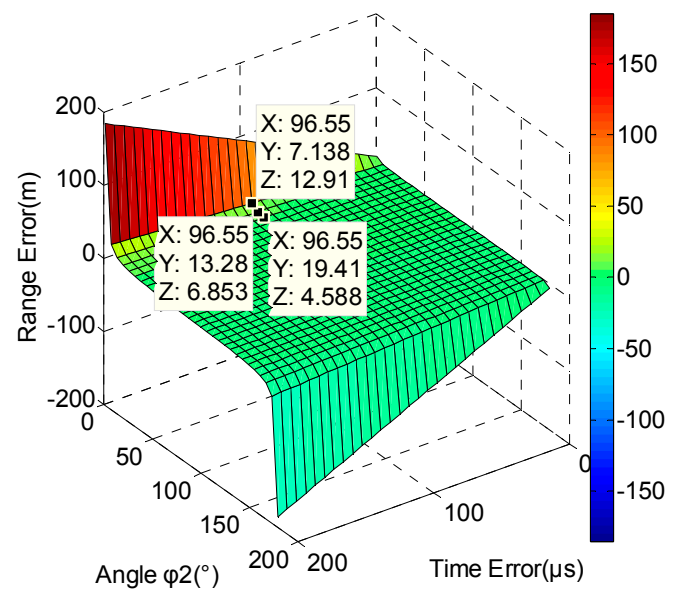

(b) $D=3 \mathrm{~m}$

Figure 5. Distributions of the range error of $R$ and $D$.

Since array distance $L$ is independent to time $t$, the error expression of $L$ is:

$$
\sigma_{R}^{L}=\frac{\partial R}{\partial L}
$$

Take partial derivatives of $L$ in Equation (24):

$$
\sigma_{R}^{L}=\frac{k_{2} \sqrt{1+k_{1}^{2}}}{2\left(k_{2}-k_{1}\right)}=\frac{k_{2} k_{1} \sqrt{1+\frac{1}{k 1^{2}}}}{2\left(k_{2}-k_{1}\right)}
$$

Substituting $\left\{\begin{array}{l}y=R \sin \varphi_{1} \\ k_{1}=\tan \varphi_{1}\end{array}\right.$ into Equation (33) gives

$$
\sigma_{R}^{L}=\frac{R}{L}
$$

Equation (34) was the expression of the range error with factor $L$. The error was affected by range $R$ and array distance $L$. The relative error is $1 / L$; therefore, the relative error theoretically stays constant when $L$ was designated. The error is below $6.67 \%$ when $L \geq 15 \mathrm{~m}$. The distribution is shown in Figure 6. 


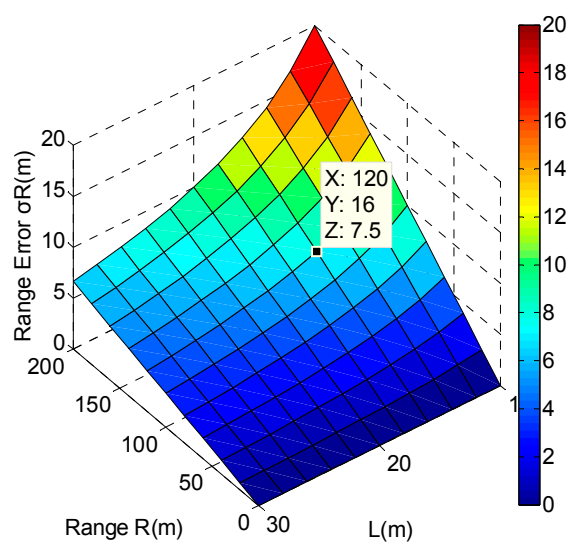

Figure 6. Distributions of the range error of $L$.

In summary, the locating ability of the double array is good. The range error stays below $5 \mathrm{~m}$ under a range condition of $100 \mathrm{~m}$ in most areas, and the azimuth error remains below $0.2^{\circ}$ for all conditions. Considering the environmental factor $(c)$ and calculated factor $\left(\sigma_{\tau}\right)$, it is advisable to choose large array sizes to improve localization precision. However, larger array sizes result in higher costs and increased complexity of the system.

\section{Experiments}

\subsection{Experiment Setting}

The locating experiments were conducted in the natural environment. The test area was open with a size of $150 \times 150 \mathrm{~m}^{2}$, and there were no tall reflectors along the boundary of the measurement domain. According to the empirical sound speed formula, the velocity of sound propagation was $343 \mathrm{~m} / \mathrm{s}$ for an air temperature of $21^{\circ} \mathrm{C}$. During the experiments, wind speed was very low and the localization range was about $100 \mathrm{~m}$, such that the influence of wind can be assumed negligible. Since it's difficult to keep an LPV going straight and travel at a constant speed, a simulated sound source with a smaller size was used to replace the vehicle noise.

The simulated source consisted of a $0.1 \mathrm{~kW}$ loudspeaker and a power amplifier. The biggest noise sources of LPV were the exhaust system and track system. The track noise was random and nonstatistical. So, the actual measurement of periodic exhaust noise was the sound signal that was collected during the running of the vehicle engine with rotating speed $r=1200 \mathrm{rpm}$. The sound signal is shown in Figure 7.
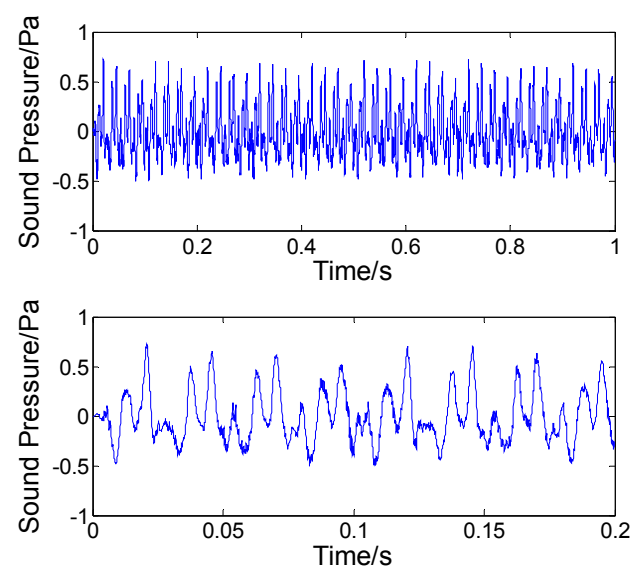

Figure 7. Sound signal for moving source. 
The moving sound source travelling at a constant speed was achieved by dragging the loudspeaker with a fixed pulley at a constant rotating speed. The source was traveling along a straight line. By monitoring the distance travelled as a function of time, the speed of motion of the source was obtained.

In Figure 8, two five-element cross arrays were set according to Figure 4 . The distance $L$ between two central sensors was $10 \mathrm{~m}$, and $2 \mathrm{~m}$ for array size $D$. A NI-PXI system with 10 channels and a sample rate of $248 \mathrm{kS} / \mathrm{s}$ was the testing instrument. The array microphones used G.R.A.S with the sensitivity of $40 \mathrm{mv} / \mathrm{pa}$.

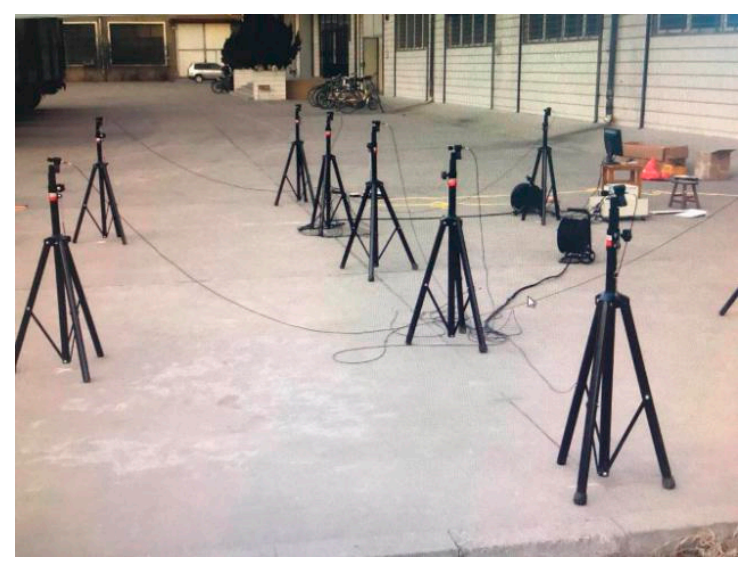

Figure 8. Microphone array.

The sound source started moving from point A $(-28.8,97.92)$ to $B(28.8,97.92)$ in the X-Y plane, and then returned back to point $\mathrm{A}$.

\subsection{Data Length for Correlation}

Since the signal collected was not even during the interval while the source moved, it is necessary to extract part of the whole signal periodically for short-time correlation. To ensure efficiency, the extraction must cover one whole period in each short-time correlation.

As the sound source was being actuated by a pulley, the speed of motion was relatively slow, which should be less than $5 \mathrm{~m} / \mathrm{s}$.

$$
f^{\prime}=\left(\frac{v_{0} \pm v_{t}}{v_{0} \mp v_{s}}\right) f
$$

According to the Doppler effect formula, the difference in value between Doppler frequency $f$ and original frequency $f$ was about $0.01 f$. Meanwhile, sound velocity $v_{0}$ was $343 \mathrm{~m} / \mathrm{s}$, velocity $v_{t}$ of the observer was zero and velocity of source $v_{s}$ took the maximum velocity $5 \mathrm{~m} / \mathrm{s}$. Therefore, data bias resulting from the Doppler effect can be ignored.

The longest distance that one acoustic wave travels in the single array is $2 D$, such that the maximum travel time is $2 \mathrm{D} / \mathrm{c}$. The sampling interval is $T_{N}=1 / F$ when the sampling frequency is assumed to be $F$. The data length $n$ for short-time correlation describes the theoretical length of each correlation in Equation (36):

$$
n \geq \frac{1 / f+2 D / c}{1 / F}
$$

\subsection{Time Compensation during Signal Transmission}

Since the acoustic signal travels a long distance before it is detected by the test system, there exists a time delay between this signal and the instant when it was emitted by the noise source. Point $x(t)$ is the position of the moving source at the instant $t$. The signal as used for the data analysis has been generated at the source at point $x\left(t_{0}\right)$, which is located at a distance $r$ from $x(t)$, as was illustrated in 
Figure 9. Thus, the identified location at the instant time $t$ is in fact the position of noise source at the moment $t_{0}$. It is essential to compensate for the difference.

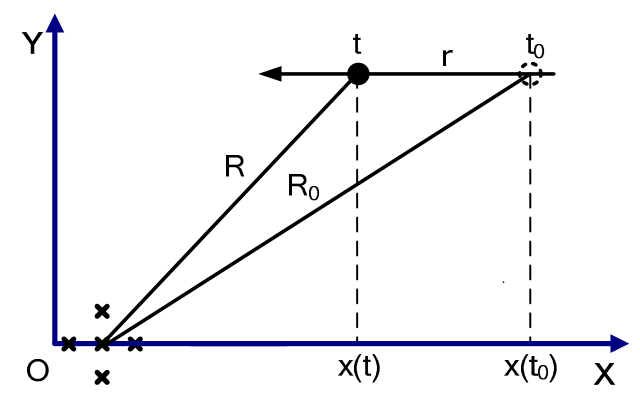

Figure 9. Path of motion of the sound source.

This constellation, as graphically illustrated in Figure 8, can be expressed as:

$$
\left\{\begin{array}{l}
R_{0}=\sqrt{x\left(t_{0}\right)^{2}+y\left(t_{0}\right)^{2}} \\
R=\sqrt{x(t)^{2}+y(t)^{2}} \\
r=\left|x\left(t_{0}\right)-x(t)\right|=v R_{0} / c
\end{array}\right.
$$

To locate the noise source in actual conditions, point $x\left(t_{0}\right)$ moves with velocity $v$ and sound velocity $c$ can be calculated. Therefore, compensation $r$ is available and needs be taken into consideration in the actual localization procedure.

\subsection{Experiment Results}

Additional environmental noise cannot be avoided either. This superposed additional noise will negatively affect the correlation of the signals. Therefore, preprocessing of the measured signals is required before obtaining the correlations. Since there were no other obvious sound sources and since the superposed additional noise is of high frequency, the wavelet-filtering method was chosen to remove unwanted noise, whereby the wavelet basis was "db10"(No. 10 of Daubechies Series Basis [27]). As the signal was relatively simple, it was decomposed into three layers. Then, the lower part in frequency was taken to perform short-time correlation. The signal-filtering process of one channel is shown in Figure 10.

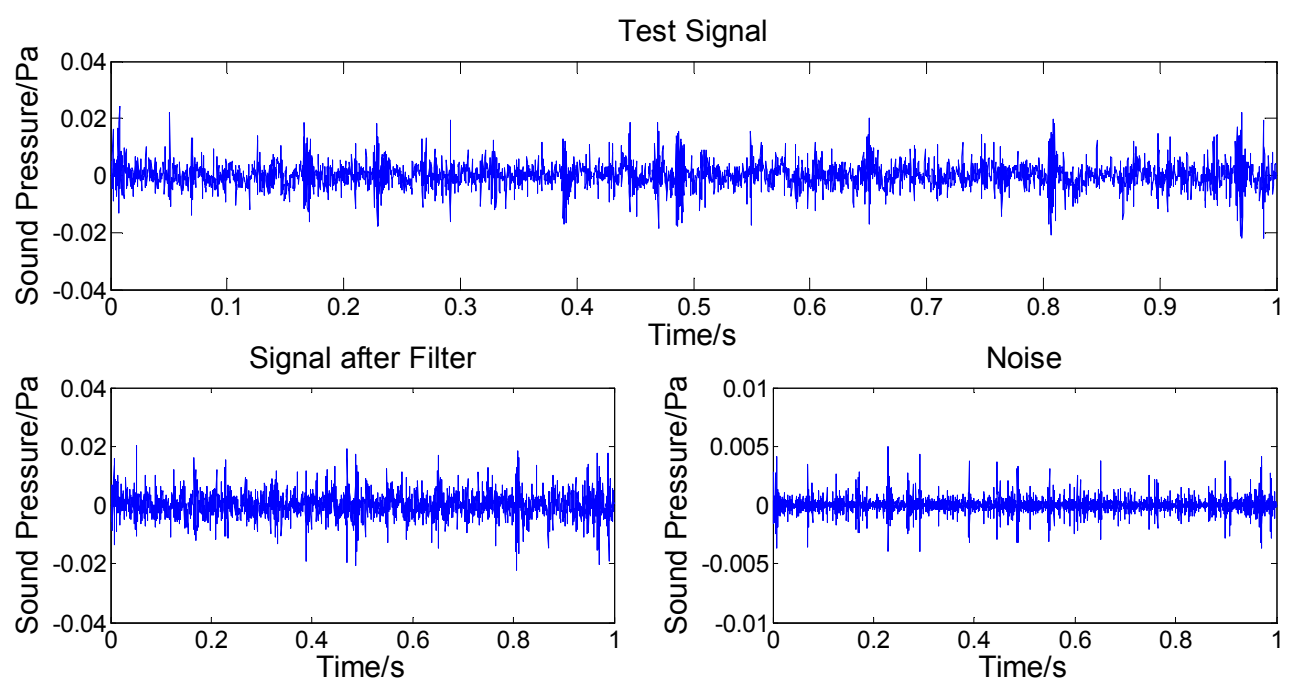

Figure 10. Filter process of signal. 
Reference to Figure 10 reveals the noise contained in the test signal. There were two kinds of noise. One is the high-frequency noise for which the amplitude is about $1 / 20$ of test signal. The other is the impulse with about $1 / 3$ of the amplitude. Both of these noise contributions detrimentally affect the correlations of the array signal. Hence, the signal form without noise, as shown in the lower left part of Figure 9, was used to operate correlations.

During the path $\mathrm{A} \rightarrow \mathrm{B}$, traveling distance was $\mathrm{S}=57.6 \mathrm{~m}$, with associated travel time $\mathrm{t}=45.6 \mathrm{~s}$ and sampling length $N=228,800$. Distance $S$ was divided into 11 elements, while travel time was the same. The localization of the moving source was achieved by locating the central point of the 11 elements. The results obtained are shown in Table 1.

Table 1. Point information of path $\mathrm{A} \rightarrow \mathrm{B}$.

\begin{tabular}{cccc}
\hline No. & Coordinates/m & Time/s & Central Point \\
\hline 1 & $(-23.56,97.92)$ & 4.15 & 20,727 \\
2 & $(-18.33,97.92)$ & 8.30 & 41,454 \\
3 & $(-13.09,97.92)$ & 12.45 & 62,181 \\
4 & $(-7.85,97.92)$ & 16.60 & 82,908 \\
5 & $(-2.62,97.92)$ & 20.75 & 103,635 \\
6 & $(2.62,97.92)$ & 24.90 & 124,362 \\
7 & $(7.85,97.92)$ & 29.05 & 145,089 \\
8 & $(13.09,97.92)$ & 33.20 & 165,816 \\
9 & $(18.33,97.92)$ & 37.35 & 186,543 \\
10 & $(23.56,97.92)$ & 41.50 & 207,270 \\
\hline
\end{tabular}

In Figure 11, it's obvious that basic frequency was $600 \mathrm{~Hz}$ with some doubling frequency component. From Section 3.2, it is known that the period of noise $T$ was $1 / f=1666 \mu$ s. Maximum traveling time of a single wave between array sensors is $2 D / c \approx 11.66 \mathrm{~ms}$. The length of signal extracted for one correlation must be bigger than $11.66 \mathrm{~ms}$. As the sampling interval was $200 \mu \mathrm{s}$, the minimum length of signal extraction was 58 .

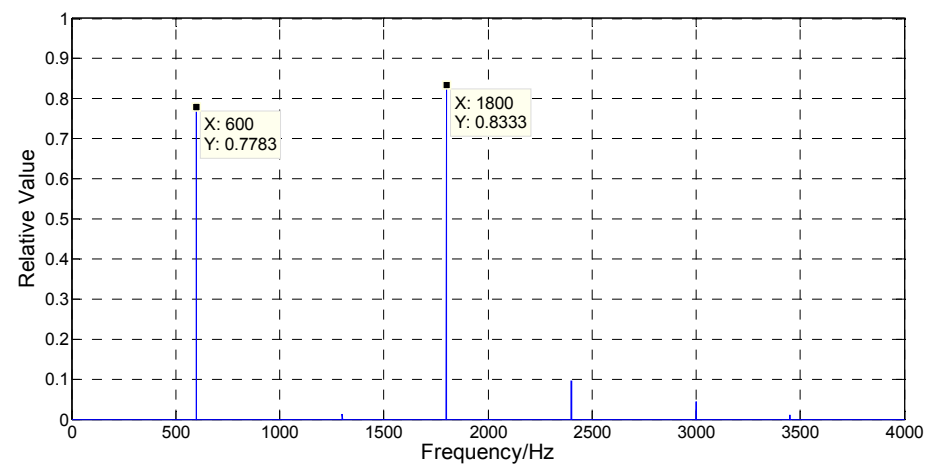

Figure 11. Frequency spectrum of the signal.

The localization was carried out at the first central point to study the choosing principle of extraction length that participated in one short-time correlation. Based on the signals of Sensor 1 and Sensor 2 in Array 1, the first central was set at point 20,727, and the length of extraction was assigned as $30,60,65,70,100,200$, and 300 . After extraction of the signal and 100 times of interpolations, the correlations of different length were calculated, as shown in Figure 12a.

The sampling interval decreased to $2 \mu$ s after 100 times of interpolation. Maximum value is located at $\mathrm{N}=3233$, while the central point of correlation was $\mathrm{N}=3000$ when extraction $n=30$. Then, time delay is $\tau_{n=30}=(3223-3000) \times 2=446 \mu$ s and delays of other lengths can be calculated in the same way, as shown in Figure 12b. 
Figure 12 illustrated that it is not possible to obtain correct time delays as time delay varies randomly when $n \leq 60$. When $n \geq 65$, delay value only varies marginally and remains steady at a level of about $520 \mu$ s, which represents the correct value of time delay.
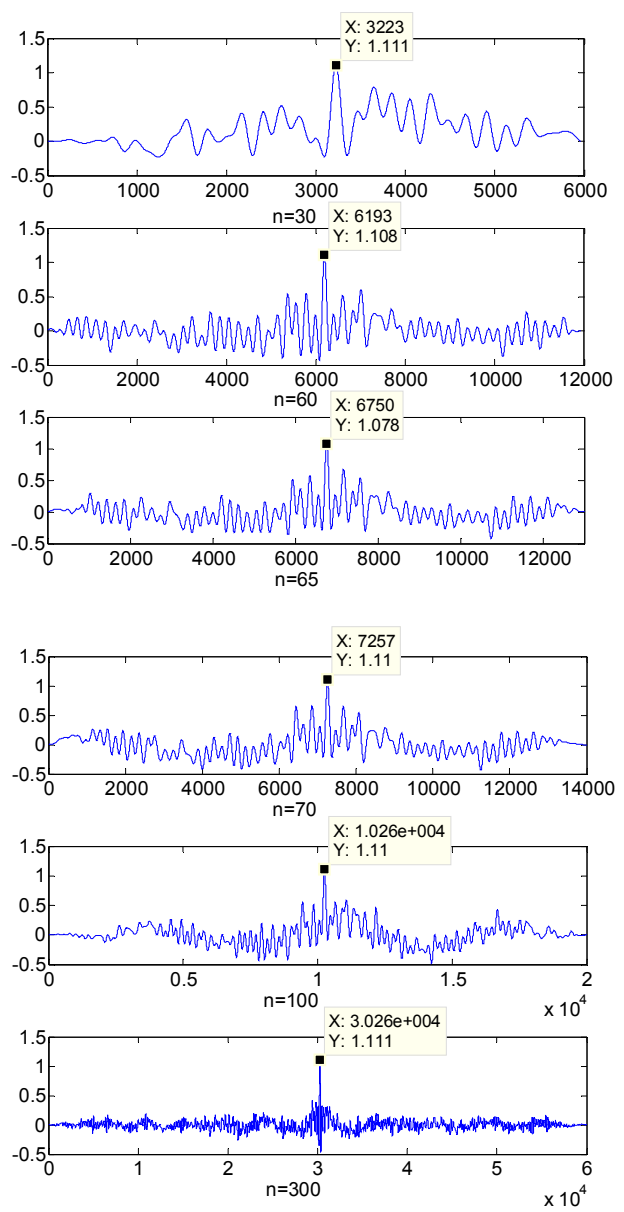

(a) Correlation Curve

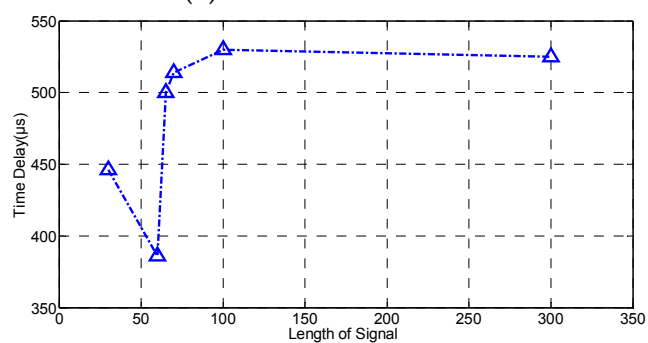

(b) Time delays

Figure 12. Correlation performance of different lengths.

The locating results of different signal lengths are shown in Table 2.

Table 2. Locating result of different lengths.

\begin{tabular}{ccc}
\hline Length & Actual Coordinates & Test Coordinates \\
\hline 30 & & $(18.24,52.31)$ \\
60 & & $(6.79,7.34)$ \\
65 & $(-23.56,97.92)$ & $(-24.36,92.95)$ \\
70 & & $(-24.35,92.70)$ \\
100 & & $(-24.20,92.80)$ \\
300 & & $(-24.07,92.17)$ \\
\hline
\end{tabular}


Table 2 illustrates that localization has failed with a rather high error when the length of the signal involved in the correlation was $n \leq 60$. When $n \geq 65$, the test point was located nearly around the actual point. Therefore, the length of the signal extraction was assigned as $n=65$, participating the calculation after interpolation.

All the locating results are summarized in Table 3, and the moving paths are shown in Figure 13.

Table 3. Locating result of moving source.

\begin{tabular}{cccc}
\hline \multirow{2}{*}{ No. } & Actual Coordinates & \multicolumn{2}{c}{ Test Coordinates/m } \\
\cline { 3 - 4 } & & $\mathbf{A} \rightarrow \mathbf{B}$ & $\mathbf{B} \rightarrow \mathbf{A}$ \\
\hline 1 & $(-23.56,97.92)$ & $(-24.01,92.82)$ & $(-23.65,98.29)$ \\
2 & $(-18.33,97.92)$ & $(-19.44,94.33)$ & $(-18.24,96.86)$ \\
3 & $(-13.09,97.92)$ & $(-14.29,94.62)$ & $(-15.85,99.70)$ \\
4 & $(-7.85,97.92)$ & $(-9.75,98.12)$ & $(-9.55,98.38)$ \\
5 & $(-2.62,97.92)$ & $(-4.58,101.67)$ & $(-3.38,98.15)$ \\
6 & $(2.62,97.92)$ & $(1.33,98.53)$ & $(2.81,96.97)$ \\
7 & $(7.85,97.92)$ & $(6.39,96.68)$ & $(8.70,97.28)$ \\
8 & $(13.09,97.92)$ & $(11.06,93.80)$ & $(13.68,95.85)$ \\
9 & $(18.33,97.92)$ & $(16.61,95.15)$ & $(19.27,96.00)$ \\
10 & $(23.56,97.92)$ & $(21.58,94.49)$ & $(25.04,95.39)$ \\
\hline
\end{tabular}

Both Table 3 and Figure 13 depict that the discrete points along the moving path were obtained accurately in the localization experiment with only small associated error.

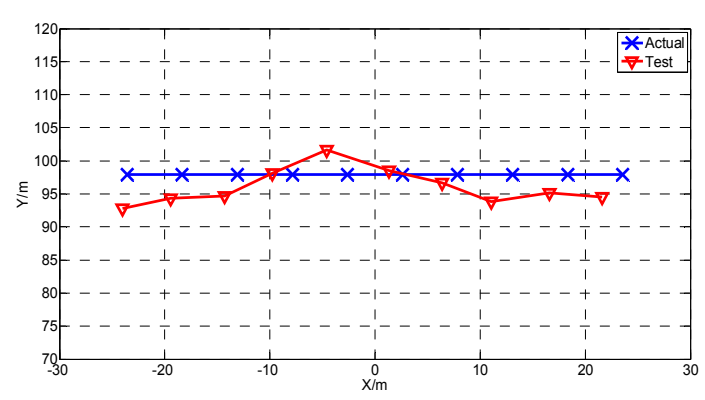

(a) $\mathrm{A} \rightarrow \mathrm{B}$

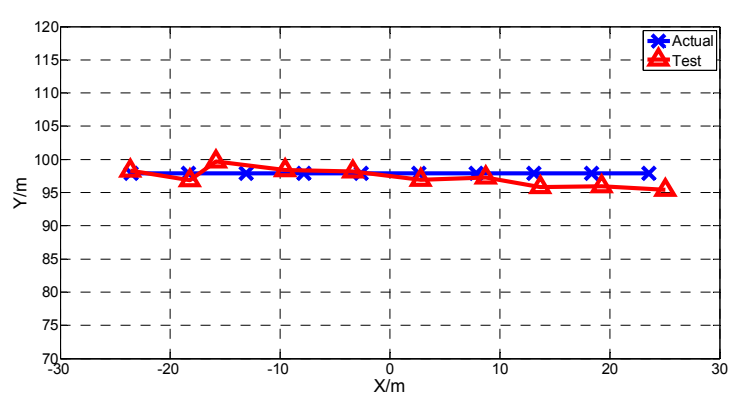

(b) $\mathrm{B} \rightarrow \mathrm{A}$

Figure 13. Actual and tested path of source.

According to Section 3.2, after compensation was considered, the relative locating error distribution is shown in Figure 14 before and after fixing.

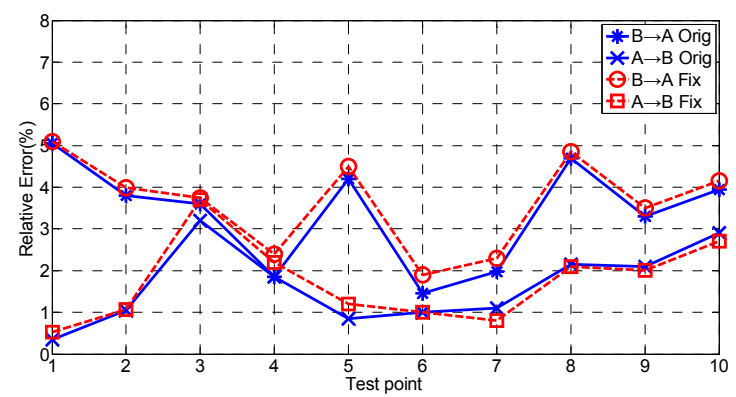

Figure 14. Distribution of relative error.

The lines in Figure 14 represent the original error of the localization, while dotted lines for error after fixing. To some extent, the locating result improved. In summary, the accurate localization 
of a moving acoustic source was achieved and the error stayed below 5\%. In further application, the complete moving path could be obtained by increasing the number of parts divided.

\section{Conclusions}

The precision analysis of a locating method of a moving sound source based on intersecting azimuth lines was studied in this paper. Simulations showed that, after another single array was added, it had better precision and lower error. The experiments were conducted outdoors after choosing principle of signal length in correlation. Accurate localization of a moving source was achieved with the associated error for locating the source staying below $5 \%$.

The work in this paper indicates applications for low-speed noise sources, such as wildlife conservation, health protection, wind turbine noise, and other engineering applications in the wild. However, the property change of the acoustic signal was ignored with low velocity and assumption of point source during the simulated source localization. Further research is required in the actual application. Further developments should focus on improvements of array size and shape. Meanwhile, the localization of high-speed moving sources and long-distance sources is not just an extension of this research.

Author Contributions: Conceptualization, J.Y. and C.X.; Methodology, C.X. and W.W.; Writing-Review \& Editing, W.W.; Supervision, Project Administration and Funding Acquisition, C.X. and W.W.

Funding: This paper is supported by the China Scholarship Council (No. 201809110025).

Conflicts of Interest: The authors declare no conflict of interest.

\section{References}

1. Muzet, A. Environmental noise, sleep and health. Sleep Med. Rev. 2007, 11, 135-142. [CrossRef] [PubMed]

2. Zacarías, F.F.; Molina, R.H.; Ancela, J.L.C.; López, S.L.; Ojembarrena, A.A. Noise exposure in preterm infants treated with respiratory support using neonatal helmets. Acta Acust. United Acust. 2013, 99, 590-597. [CrossRef]

3. Babisch, W.; Beule, B.; Schust, M.; Kersten, N.; Ising, H. Traffic noise and risk of myocardial infarction. Epidemiology 2005, 16, 33-40. [CrossRef] [PubMed]

4. Miedema, H.; Oudshoorn, C. Annoyance from transportation noise: Relationships with exposure metrics DNL and DENL and their confidence intervals. Environ. Health Perspect. 2001, 109, 409-416. [CrossRef] [PubMed]

5. Cueto, J.L.; Petrovici, A.M.; Hernández, R.; Fernández, F. Analysis of the Impact of Bus Signal Priority on Urban Noise. Acta Acust. United Acust. 2017, 103, 561-573. [CrossRef]

6. Morley, D.W.; Hoogh, K.; Fecht, D.; Fabbri, F.; Bell, M.; Goodman, P.S.; Elliott, P.; Hodgson, S.; Hansell, A.L.; Gulliver, G. International scale implementation of the CNOSSOS-EU road traffic noise prediction model for epidemiological studies. Environ. Pollut. 2015, 206, 332-341. [CrossRef] [PubMed]

7. Ruiz-Padillo, A.; Ruiz, D.P.; Torija, A.J.; Ramos-Ridaob, Á. Selection of suitable alternatives to reduce the environmental impact of road traffic noise using a fuzzy multi-criteria decision model. Environ. Impact Assess. Rev. 2016, 61, 8-18. [CrossRef]

8. Licitra, G.; Fredianelli, L.; Petri, D.; Vigotti, M.A. Annoyance evaluation due to overall railway noise and vibration in Pisa urban areas. Sci. Total Environ. 2016, 568, 1315-1325. [CrossRef] [PubMed]

9. Bunn, F.; Trombetta, Z.P.H. Assessment of railway noise in an urban setting. Appl. Acoust. 2016, 104, 16-23. [CrossRef]

10. Paolo, G.; Luca, F.; Duccio, S.; Gaetano, L. ADS-B System as a Useful Tool for Testing and Redrawing Noise Management Strategies at Pisa Airport. Acta Acust. United Acust. 2017, 103, 543-551.

11. Iglesias-Merchan, C.; Luis, D.; Mario, S. Transportation planning and quiet natural areas preservation: Aircraft overflights noise assessment in a National Park. Transp. Res. Part Transp. Environ. 2015, 41, 1-12. [CrossRef] 
12. Kephalopoulos, S.; Paviotti, K.; Maercke, D.V.; Shilton, S.; Jones, N. Advances in the development of common noise assessment methods in Europe: The CNOSSOS-EU framework for strategic environmental noise mapping. Sci. Total Environ. 2014, 482, 400-410. [CrossRef] [PubMed]

13. Morel, J.; Marquis-Favre, C.; Gille, L.A. Noise annoyance assessment of various urban road vehicle pass-by noises in isolation and combined with industrial noise: A laboratory study. Appl. Acoust. 2016, 101, 47-57. [CrossRef]

14. Sakhaeifar, M.; Banihashemrad, A.; Liao, G.Y.; Waller, B. Tyre-pavement interaction noise levels related to pavement surface characteristics. Road Mater. Pavement Des. 2018, 5, 1044-1056. [CrossRef]

15. Donavan, P.R. The effect of pavement type on low speed light vehicle noise emission. SAE Tech. Pap. 2005, 1, 2416.

16. Gille, L.A.; Marquis-Favre, C.; Morel, J. Testing of the European Union exposure-response relationships and annoyance equivalents model for annoyance due to transportation noises: The need of revised exposure-response relationships and annoyance equivalents model. Environ. Int. 2016, 94, 83-94. [CrossRef] [PubMed]

17. Janssen, S.A.; Vos, H.; Eisses, A.R.; Pedersen, E. A comparison between exposure-response relationships for wind turbine annoyance and annoyance due to other noise sources. J. Acoust. Soc. Am. 2011, 130, 3743-3756. [CrossRef] [PubMed]

18. Gallo, P.; Fredianelli, L.; Palazzuoli, D.; Licitra, G.; Fidecaro, F. A procedure for the assessment of wind turbine noise. Appl. Acoust. 2016, 114, 213-217. [CrossRef]

19. Schulte-Werning, B.; Jäger, K.; Strube, R.; Willenbrink, L. Recent developments in noise research at Deutsche Bahn. J. Sound Vib. 2003, 267, 689-699. [CrossRef]

20. Park, S.H.; Kim, Y.H. An improved moving frame acoustic holography for coherent band limited noise. Acoust. Soc. Am. 1998, 104, 3179-3189. [CrossRef]

21. Buckingham, M.J. On the sound field from a moving source in a viscous medium. Acoust. Soc. Am. 2003, 11, 3112-3118. [CrossRef]

22. Park, S.H.; Kim, Y.H. Visualization of pass by noise by means of moving frame acoustic holography. J. Acoust. Soc. Am. 2001, 109, 2326-2339. [CrossRef]

23. Park, C.S.; Kim, Y.H. Time domain visualization using acoustic holography implement by temporal and spatial complex envelope. J. Acoust. Soc. Am. 2009, 126, 1659-1662. [CrossRef] [PubMed]

24. Boone, M.M.; Kinneging, N.; Dool, T.V. Two-dimensional Noise Source Imaging with T-shaped Microphone Cross Array. J. Acoust. Soc. Am. 2000, 108, 2884-2890. [CrossRef]

25. Si, C.D.; Chen, E.L.; Yang, S.P.; Wang, C.Y. Experimental study on noise sources identification of vehicle based on microphone array technology. J. Vib. Shock 2009, 28, 173-175.

26. Wang, W.J.; Thomas, P.J. Low-frequency active noise control of an underwater large-scale structure with distributed giant magnetostrictive actuators. Sens. Actuators A Phys. 2017, 263, 113-121. [CrossRef]

27. Ingrid, D. Ten Lectures on Wavelets; Society for Industrial and Applied Mathematics: Philadelphia, PA, USA, 1992. 